\title{
Ergonomía aplicada a las tareas del hogar
}

Figueroa Valenzuela, María Eugenia

\begin{abstract}
- Abstract
Home activities demand important efforts and they are accentuated when the people have some disease of musculoskeletal system. The principles and tools of ergonomics, used in this article at home activities, allows to Occupational Therapists to take part on the physical environment, decreasing its exigencies for the control of injuries risks and of improving the performance of persons with physical disabilitys.
\end{abstract}

\section{Resumen}

Las tareas del hogar demandan esfuerzos importantes, que se acentúan en el caso de estar afectado por alguna enfermedad del sistema músculo-esquelético. Aplicar los principios y herramientas de la ergonomía ejemplificados en este artículo a las tareas del hogar, permite a los Terapeutas Ocupacionales intervenir sobre el entorno físico, aminorando sus exigencias para controlar riesgos de lesiones y mejorar el desempeño de esta actividad, especialmente en personas con discapacidad física.

Terapeuta Ocupacional. Licenciada en Ciencia de la Ocupación. Másteren Ergonomía. Universidad Politécnica de Cataluña. Profesor Asitente de la Escuela de Terapia Ocupacional de la Facultad de Medicina de la Universidad de Chile.Contacto > > mefigueroa@biomecanica.cl 


\section{- Introducción}

La ergonomía es una disciplina que se aplica en la actualidad a todos los ámbitos de la actividad humana, preocupándose de mejorar la interacción de los individuos con su entorno físico. Una relación inadecuada produce en las personas sobrecargas físicas o mentales, que en situaciones extremas, pueden llegar a causar serias enfermedades.

Para la Terapia Ocupacional el manejo de herramientas ergonómicas es fundamental, ya que las personas enfermas o con alguna discapacidad, presentan una relación con el entorno más precaria, por estar éste diseñado, por lo general, para personas sanas.

La Ergonomía utiliza en su práctica, conocimientos generados por otras ciencias y disciplinas que se ocupan del individuo, como las ciencias de la salud, la psicología y la sociología. Para analizar los riesgos ergonómicos de las tareas del hogar, motivo de este artículo, se utilizará un enfoque biomecánico que permite identificar aspectos que pueden constituir un riesgo para el sistema músculo-esquelético y que son particularmente importantes de abordar por los Terapeutas Ocupacionales, especialmente en el tratamiento de personas que sufren tanto enfermedades crónicas como artritis reumatoide o artrosis, como enfermedades agudas, como lumbalgias o tendinitis, entre otras.

Las tareas del hogar son una parte importante de las actividades de la vida diaria de muchas personas, y en nuestra cultura, como en otras, forman parte del rol inherente a la mujer. Además esta actividad, constituye la fuente de trabajo para más de 500.000 mujeres en nuestro país (1) lo que las hace más relevantes.

En el análisis biomecánico se estudia el cuerpo humano como si se tratara de un sistema mecánico, analizando la forma en que el organismo ejerce fuerza y genera movimiento y se basa principalmente en la anatomía, la fisiología y la física, para comprender por qué algunas tareas provocan daños o enfermedades. Debe aclararse que se utiliza este enfoque por fines prácticos y por su pertinencia, pero reconociendo sus limitaciones para el estudio de la actividad humana, cuyo estudio requiere además, la consideración de aspectos como la motivación y los intereses, lo que resulta absolutamente indispensable.

Desde una perspectiva física, todas las estructuras responden a la aplicación de fuerzas con una deformación que está limitada a la resistencia del material, de esta manera, existe una relación entre la resistencia del material y la magnitud de la fuerza necesaria para deformarlo. Estos principios tan evidentes en los materiales, son aplicables a las distintas estructuras corporales, sin embargo, en el cuerpo humano aún no se ha podido determinar con precisión los límites físicos de ligamentos, tendones, discos intervertebrales, cápsulas articulares, músculos y otras estructuras involucradas en el movimiento. Esta dificultad deriva de que los límites varían por motivos tan diversos como la predisposición genética, la edad o los esfuerzos acumulativos.

Esta complejidad ha sido abordada por la biomecánica, identificando las condiciones en que los movimientos someten -a las diferentes estructuras corporales- a niveles de tensión que aumentan la probabilidad de producir lesiones. Estas condiciones son identificadas como sobreesfuerzos o sobrecarga y se explican mediante el efecto que producen determinadas posturas, la manipulación de cargas, la frecuencia con que se realizan los movimientos o la combinación de estos factores sobre determinados segmentos o estructuras corporales. Estas condiciones son identificadas en Ergonomía, como factores de riesgo y constituyen el foco de análisis. 


\section{Tareas que requieren el uso de fuerza}

La necesidad de utilizar la fuerza está relacionada con el hecho de tener que mover objetos y herramientas, o tener que mantener el cuerpo en una postura determinada. Esta exigencia está presente en muchas tareas domésticas como: mover muebles, cargar ollas, hacer las compras o atender a un bebé, todas estas tareas son potencialmente peligrosas tanto para la columna vertebral como para las extremidades superiores.

Debido a la frecuencia con que se presentan afecciones a la columna vertebral, comenzaremos por explicar las condiciones que ponen en riesgo a esta estructura. Los estudios biomecánicos han demostrado que existe un límite seguro en el peso para la manipulación. Sobre este límite, se expone al disco intervertebral $L_{5}-S_{1}$ a sufrir una lesión. Este límite se ha establecido en $23 \mathrm{Kg}$, pero es aplicable sólo si se cumplen determinadas condiciones para la manipulación como: mantener la carga pagada al cuerpo y a la altura de la cintura, no realizar giros mientras se sostiene la carga, que esté sostenida de forma segura y que la frecuencia de la manipulación no sea muy elevada. Si algunas de estas condiciones no se encuentran presenten, el límite de 23 Kg., disminuye.

A modo de ejemplo, se calculará el índice de levantamiento, según el método de la ecuación $\mathrm{NIOSH}$ (2), para estimar el riesgo al trasladar a un niño pequeño (de $10 \mathrm{Kg}$.) dormido, desde la cama de su madre a su cuna. (Figura 1)

- Peso del niño: $10 \mathrm{Kg}$

- Altura de la cama normal: $45 \mathrm{cms}$

- Altura de la baranda de la cuna: $80 \mathrm{~cm}$

- Desplazamiento horizontal: $47 \mathrm{~cm}$ (distancia desde el centro de gravedad de la madre al del niño al dejarlo en la cuna)

- Desplazamiento vertical: $35 \mathrm{~cm}$ (distancia desde la cama a la altura de la baranda y desde la baranda a la cuna)

- Seguridad en el agarre: Buena

- Giro de columna: Sí

- Frecuencia: Muy baja (considerando sólo una vez) 


\section{Figura 1}

Riesgo de lesiones en la columna

por manipulación de un bebe

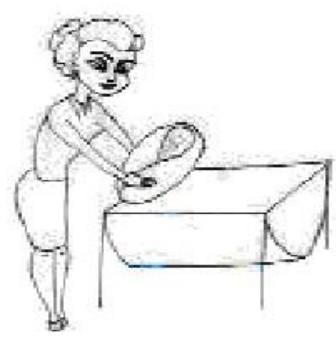

Cada una de las condiciones de manipulación determina un factor, que corrige el límite de peso aceptable aplicando la siguiente ecuación:

$$
L C \times H M \times V M \times D M \times A M \times F M \times C M=R W L
$$

donde:

LC, es "Límite de Carga" (23Kg)

HM, es el factor "Multiplicador Horizontal"

VM, es el factor "Multiplicador Vertical"

DM, es el factor "Multiplicador de Distancia"

FM, es el factor "Multiplicador de Frecuencia"

AM, es el factor "Multiplicador de Asimetría"

$\mathrm{CM}$, es el factor "Multiplicador de Acoplamiento"

RWL, es el "Límite de Peso Recomendado"

Con las condiciones señaladas el peso recomendado sería de: $7.2 \mathrm{Kgs}$.

Con un Índice de Levantamiento (peso real/peso recomendado) para esta situación de: 1.3, lo que quiere decir que en la situación planteada se supera en un $30 \%$ lo recomendado, presentando una importante exposición a sufrir lesiones lumbares.

Esta herramienta biomecánica, permite por un lado demostrar la importancia de las condiciones de manipulación y por otro, estimar el nivel de riesgo de lesiones a la columna vertebral. Lo mismo puede aplicarse a tareas aparentemente inofensivas como cargar una olla desde la cocina al lavaplatos, para escurrir el agua de cocción o transportar bolsas del supermercado. 
En el primer ejemplo, la utilización de barandas deslizables que permitan acercarse a la cuna y evitan la manipulación en una posición de alto riesgo, sería la intervención más efectiva. Así como, en el ejemplo de la olla la utilización de una herramienta para retirar su contenido y vaciar la olla cuando esté fría y pueda manipularse pegada al cuerpo, sería lo más recomendable.

Insistir en dirigir la mirada sobre los aspectos del entorno físico responsables de que una tarea se convierta en una situación de riesgo, tiene la intensión de aclarar que muchas interpretaciones que responsabilizan al paciente "por sus malas prácticas", están justificadas por diseños inadecuados del entono y recomendaciones livianas. Un ejemplo es la creencia que manipular una carga desde el piso, flectando las rodillas y manteniendo la columna erguida, es una medida suficiente para no sufrir lesiones a la columna y lo cierto, es que se ha demostrado que en esa situación sólo sería seguro manipular, para una persona sana, una carga que no sobrepase los $14 \mathrm{~kg}$. Por este motivo, estas recomendaciones generales, utilizadas livianamente en muchos casos, son una invitación a sobrepasar las capacidades fisiológicas y exponen a mayor riesgo.

\section{Posiciones recomendadas según las exigencias de la tarea}

Algunas tareas del hogar como planchar o cocinar, se realizan preferentemente de pie y su duración, en algunas ocasiones, puede ser bastante prolongada. Esta posición puede producir fatiga por el esfuerzo muscular utilizado para bloquear las articulaciones de caderas y rodillas y produce una disminución del retorno venoso, lo que acentúa algunos trastornos circulatorios en los miembros inferiores. Sin embargo, en esta posición el cuerpo tiene una gran movilidad y previene las tensiones que aparecen en la columna y extremidades superiores al intentar alcanzar objetos distantes, desde la posición sentado. Por lo tanto, se recomienda estar de pie cuando las tareas exijan movimientos amplios y/o la manipulación de objetos medianos o grandes, como planchado de sábanas o lavado de platos, y estar sentado para realizar tareas finas o de precisión, como picar o pelar verduras y planchar piezas pequeñas o delicadas, ya que en la posición sentado se reduce el gasto energético, disminuye la fatiga y se tiene mayor control de los movimientos (precisión), pero debe considerarse que estas recomendaciones son generales y no han considerado la condición física de quien las realiza.

Al no existir una regla que permita recomendar una posición u otra, sólo el análisis de las exigencias físicas de cada tarea y de las capacidades o limitaciones de quienes las realizan, determinan finalmente la posición más recomendada.

La Ergonomía propone la alternancia de posiciones como principal estrategia para atenuar los riesgos de una posición en particular, lo que se obtiene fraccionando las tareas largas, de exigencias físicas similares e intercalando tareas que demanden la acción de otros grupos musculares. Algunas adaptaciones del entorno que facilitan esta alternancia es disponer asientos o pisos en la cocina u otros lugares que lo requieran, y adecuar las superficies de trabajo a la altura de los usuarios considerando las tareas que realizan. Por ejemplo, la tabla de planchar debe ajustarse de modo que el mango de la plancha, quede aproximadamente al nivel de la cintura de la persona, lo que puede lograrse tanto de pie como sentado como se observa en la figura 2, o adecuando una zona de la cocina para la ejecución de tareas sentado con el plano de trabajo a la altura de los codos. Estas adaptaciones pueden ser de gran utilidad para personas que sufren enfermedades con trastornos circulatorios en las extremidades inferiores, como várices, o baja tolerancia a la posición de pie, como ancianos o personas en etapa de recuperación de reposos prolongados. 
Figura 2

Adecuación de superficie de trabajo para tareas sentado y de pie
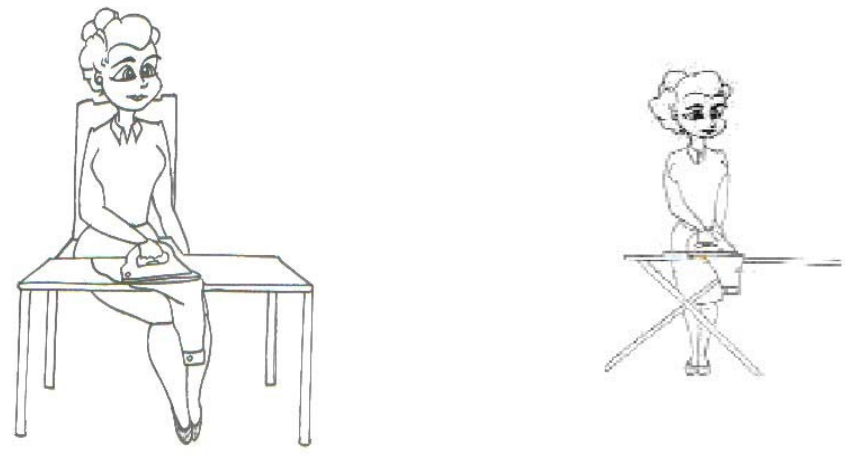

\section{Riesgos posturales en los distintos segmentos}

La carga estática producida por las posturas forzadas, se puede observar en cualquier segmento corporal, cuando las articulaciones se alejan de la posición neutra. En estos casos se genera una contracción muscular local y la consecuente fatiga, que cuando es prolongada puede llegar a provocar trastornos o patologías. El riesgo aumenta cuanto más forzada es la postura y cuanto menor es el número de apoyos. Algunas tareas que presentan este factor de riesgo son: limpiar una tina, lavar vidrios, ordenar la parte superior de closet o de muebles de cocina y sacudir en altura.

La sobrecarga postural, ya sea de las extremidades superiores o la columna, debe evaluarse asociada al tiempo de exposición, pero en términos generales, deben evitarse las posturas que exijan mantener los brazos en rangos que superen los $45^{\circ}$ de flexión en hombros y la flexión de columna, en cualquier rango, ya que su musculatura se fatiga rápidamente. Una tarea habitual, que mantiene la espalda en flexión por varios minutos, es el lavado de platos, como se observa en la fig. 3, donde el riesgo esta determinado por la altura inadecuada del plano de trabajo. Por este motivo además, el riesgo aumenta cuanto más alto es el usuario o más prolongado es el tiempo de lavado.

Estas posturas son responsables del desarrollo de procesos inflamatorios de tejidos blandos en hombros y columna, y acentúan dolencias como lumbagos, cervicalgias o síndromes dolorosos de las extremidades superiores. 


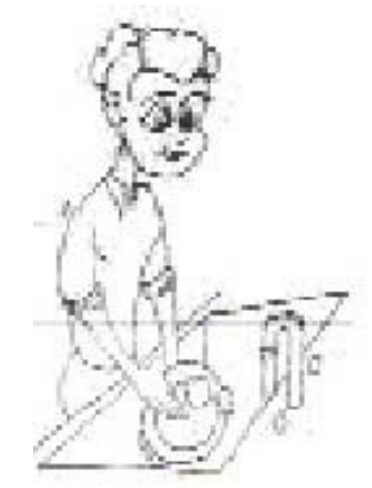

Figura 3

Sobrecarga postural por plano de trabajos inclinados

Las intervenciones para controlar los riesgos posturales son la corrección de la altura del plano de trabajo, considerando las características antropométricas de los usuarios y la implementación de herramientas, como limpiadores con mango (mopas), que permiten lavar tinas y azulejos desde una posición segura. En forma complementaria, se puede intervenir sobre la organización de las rutinas de trabajo, como por ejemplo, no concentrando las tareas con sobrecarga postural en un día, como el lavado de vidrios que genera importantes niveles de fatiga.

\section{- La frecuencia de los movimientos}

La repetición es un factor de riesgo, que asociado a los tratados anteriormente: manipulación de cargas o sobrecarga postural, representa una de las principales causas de inflamación de tendones e inserciones musculares. A diferencia de lo que sucede en el ámbito laboral, donde las tareas asignadas están muy definidas y los patrones de movimiento se repiten constantemente, en el hogar las tareas son múltiples y variadas, demandando esfuerzos que involucran distintas estructuras y grupos musculares, lo que funciona como factor protector. Pese a esto, el factor repetitividad se observa en algunas ocasiones vinculado al cuidado de los niños, por ejemplo, cuando los bebes deben ser mudados o cuando están aprendiendo a controlar esfínteres. En estos casos las medidas ergonómicas juegan un rol importante a la hora de prevenir dolencias físicas y son fundamentales en personas con cuadros inflamatorios crónicos, como enfermedades del colágeno.

En estos casos, las intervenciones orientadas al cuidado de posturas mediante la implementación de áreas de trabajo seguras, con planos de trabajo ajustables o adecuados a las características antropométricas, y proporcionar elementos de soporte, como apoyabrazos o mesas con escotaduras, deben realizarse tempranamente.

\section{Evaluación del entorno físico}

Como se ha señalado, las tareas del hogar exigen una serie de capacidades, que podrían estar disminuidas en muchas enfermedades que comprometen al sistema músculo-esquelético, como cuadros que cursan con debilidad muscular, rigidez articular, inflamación de las articulaciones 0 estructuras periarticulares, entre otras. 
Para abordar la intervención del entorno físico es necesario realizar, en primer lugar, una evaluación de la capacidad funcional del paciente, considerando su movilidad articular, fuerza y tolerancia al esfuerzo para, a continuación, realizar un análisis de las actividades de hogar en forma rigurosa y en situación real, detectando las tareas que generan dolor y/o determinan mayor esfuerzo. Esta evaluación servirá para identificar las tareas críticas y diseñar la intervención ergonómica.

Es importante que la evaluación se realice en la casa del paciente, analizando las tareas que forman parte su rutina diaria. Lamentablemente, las entrevistas y las salas de AVD, no sirven para esta finalidad, ya que no pueden simular las condiciones reales, detectar los verdaderos obstáculos e identificar las distintas estrategias que la persona utiliza cuando se encuentra en su medio.

Los principales aspectos a evaluar en el análisis de la actividad son:

1. Presencia de dolor: Para esto se deben identificar además de las tareas, las operaciones específicas dentro de cada tarea que producen molestias o dolor. Se recomienda el uso de la Escala Análoga, donde el paciente asigna un nivel de 1 a 10 a su percepción del dolor durante la ejecución de la actividad.

Con esta información se construye una tabla que correlaciona la presencia y nivel de dolor con las tareas y operaciones que lo desencadenan. Junto a cada operación se registran los factores de riesgo asociados: movimientos, postura, fuerza y tiempo de exposición.

2. Medición del esfuerzo: Para evaluar el esfuerzo involucrado en el desempeño de cada tarea y sus operaciones, se recomienda para esto utilizar una herramienta que valora los esfuerzos y los correlaciona con signos observables en el paciente, como expresiones gestuales, se ejemplifica en la tabla 1.

\section{Tabla 1: Determinación de niveles de esfuerzo. Adaptación de escala de Borg ${ }^{(3)}$}

\begin{tabular}{|l|c|l|}
\hline $\begin{array}{c}\text { Calificación del } \\
\text { esfuerzo }\end{array}$ & $\begin{array}{c}\text { Niveles de } \\
\text { esfuerzo }\end{array}$ & \multicolumn{1}{|c|}{ Esfuerzo percibido } \\
\hline Liviano & $1-2$ & Relajado. No se aprecia esfuerzo \\
\hline Algo pesado & 3 & $\begin{array}{l}\text { Esfuerzo claro. Se percibe levemente en expresión } \\
\text { facial }\end{array}$ \\
\hline \hline Pesado & $4-5$ & $\begin{array}{l}\text { Esfuerzo evidente. Se percibe claramente en la } \\
\text { expresión facial }\end{array}$ \\
\hline \hline Muy pesado & $6-7$ & $\begin{array}{l}\text { Esfuerzo sustancial.se agrega a la expresión facial } \\
\text { movimientos corporales }\end{array}$ \\
\hline Casi Máximo & 7 & $\begin{array}{l}\text { Uso de cuerpo completo, involucra hombros y tronco } \\
\text { para hacer esfuerzo. Se perciben cambios en el ritmo } \\
\text { respiratorio }\end{array}$ \\
\hline
\end{tabular}

Este análisis de la actividad, consignando niveles de dolor y esfuerzo, determina las tareas críticas y focaliza la intervención ergonómica a las actividades de mayor riesgo para el paciente.

La ejecución de ninguna tarea debe producir dolor o sobrepasar un nivel de esfuerzo 3, por lo que la intervención ergonómica se realiza modificando los aspectos del entorno físico que generan exigencias, como posturas forzadas, movimientos sobre los rangos de confort, manipulación de pesos, etc. El análisis de la actividad debe realizarse nuevamente, al implementar la intervención ergonómica, para medir su efectividad. 
En Ergonomía, la intervención sobre el entorno físico debe procurar, dependiendo de la disponibilidad económica, que las adaptaciones sean las más normales posibles. Prefiriendo productos comunes y disponibles en el mercado, por sobre a las adaptaciones artesanales, que marcan diferencias evidentes entre las personas sanas y las enfermas.

Un ejemplo ilustrativo podría ser la intervención en casas de pacientes artríticos, donde es preferible remplazar las llaves de agua de cierre giratorio (que aumentan la desviación cubital de los dedos), por llaves de palanca, que se utilizan con frecuencia en las edificaciones modernas. Esta intervención es muy bien recibida tanto por el paciente como por su familia, y tiene mejores resultados que educar en el uso de técnicas de protección articular o la instalación de adaptaciones poco estéticas.

\section{- Conclusión}

La vida moderna ha simplificado las tareas del hogar, sin embargo, algunas tareas siguen demandando esfuerzos importantes y exponiendo a riesgos, especialmente a personas padecen alguna enfermedad del sistema músculo-esquelético.

El logro de condiciones ergonómicas en las tareas del hogar es posible, sólo requiere una evaluación de las capacidades funcionales de los pacientes, que determine en forma objetiva sus limitaciones y un análisis de la actividad, realizado en las tareas habituales, en condición real. Estas evaluaciones permiten valorar no solamente el nivel de dependencia o independencia, sino también los niveles de esfuerzo y riesgos involucrados en el desempeño de las tareas.

El procedimiento sugerido, identificando las tareas u operaciones que producen dolor o fatiga, es una estrategia complementaria al enfoque tradicional de la rehabilitación, centrado en la recuperación de las capacidades funcionales y que resulta altamente eficaz para prevenir el deterioro de las capacidades físicas, mejora los niveles de independencia y en personas que desempeñan dentro de su rutina tareas del hogar, permite además, el ejercicio de un rol, que para muchas mujeres es primordial.

La intervención sobre las exigencias del entorno físico, aplicando herramientas ergonómicas, es aplicable a los distintos ámbitos de la actividad humana, posibilitando a algunas personas el desempeño satisfactorio y un mayor nivel de autonomía, en otras actividades como el trabajo o el estudio, que sin este abordaje no podrían ser desarrolladas. 
- Referencias

MOORE J.S, GARG A The Strain Index: A Proposed Method to Analyze Jobs For Risk of Distal Upper Extremity Disorders., 1995; AlHA Journal, 56(5): 443-458.

- AYOUB M. Control of Manual Lifting Hazards: III. Preemploymen Screening. Journal of Occupational Medicine, 1982. 10 (24): 751-761.

- CHAVARRIA R La carga física de trabajo: definición y evaluación.

Notas Técnicas de Prevención INSHT, 1991. NTP 295: 1-6.

(1) EVANS E Conferencia en encuentro del INP con Asesoras del Hogar, Dic. 2003 (no publicado).

(2) WATERS T, PUTZ-ANDERSON V, GARG A. Applications Manual for the Revised NIOSH Lifting Equation National Institute for Occupational Safety and Health, January 1994 (DHHS, NIOSH Publication No. 94-110). 